\title{
Polydispersity-induced macrophase separation in diblock copolymer melts
}

Article

Published Version

Matsen, M. W. (2007) Polydispersity-induced macrophase separation in diblock copolymer melts. Physical Review Letters, 99. p. 148304. doi:

https://doi.org/10.1103/PhysRevLett.99.148304 Available at https://centaur.reading.ac.uk/769/

It is advisable to refer to the publisher's version if you intend to cite from the work. See Guidance on citing.

Published version at: http://dx.doi.org/10.1103/PhysRevLett.99.148304

To link to this article DOI: http://dx.doi.org/10.1103/PhysRevLett.99.148304

All outputs in CentAUR are protected by Intellectual Property Rights law, including copyright law. Copyright and IPR is retained by the creators or other copyright holders. Terms and conditions for use of this material are defined in the End User Agreement.

\section{www.reading.ac.uk/centaur}

\section{CentAUR}

Central Archive at the University of Reading

Reading's research outputs online 


\title{
Polydispersity-Induced Macrophase Separation in Diblock Copolymer Melts
}

\author{
M. W. Matsen \\ Department of Mathematics, University of Reading, Whiteknights, Reading RG6 6AX, United Kingdom
}

(Received 28 June 2007; published 5 October 2007)

\begin{abstract}
The effect of $A$-block polydispersity on the phase behavior of $A B$-diblock copolymer melts is examined using a complete self-consistent field theory treatment that allows for fractionation of the parent molecular-weight distribution. In addition to observing the established shift in phase boundaries, we find the emergence of significant two-phase coexistence regions causing, for instance, the disappearance of the complex phase window. Furthermore, we find evidence that polydispersity relieves packing frustration, which will reduce the tendency for long-range order.
\end{abstract}

In complex liquids, involving, for example, polymers, colloids, or liquid crystals, the constituents come in a distribution of sizes, which generally complicates their equilibrium phase behavior [1]. Although this difficulty can be ignored if sufficient care is taken to keep the distribution narrow, it can be expensive and time consuming to produce such model materials. Thus there is good reason to "bite the bullet" and study the effects of polydispersity, especially since it may actually result in advantageous behavior. The most challenging aspect is the treatment of multiphase coexistence regions. Much of the effort and theoretical techniques for macrophase separation [2] have been restricted to uniform phases characteristic of simple liquids such as polymer solutions or blends. Complex liquids have received far less attention, and most of that has been in regards to the nematic and smectic phases exhibited by thermotropic liquid crystals [3]. The lyotropic liquid crystalline behavior of lipid, surfactant, block-copolymer systems has been relatively neglected until very recently. New activity involving blockcopolymer melts has been spurred on by economical synthetic techniques $[4,5]$, which would make these materials far more attractive to the manufacturing industry provided that the high levels of polydispersity do not compromise their novel properties [6]. The most serious concern is whether or not macrophase separation prevents the formation of well-ordered morphologies; monodisperse melts are exempt from two-phase separation because there is only a single molecular species. For a while, there was little experimental evidence for macrophase separation $[5,7,8]$, but observations of two-phase coexistence are becoming increasingly common [4,9-11]. Although a couple of theoretical calculations $[12,13]$ have explored polydispersity effects on the phase diagram, the issue of macrophase separation has, so far, been neglected. Thanks to new computational developments $[14,15]$, we are now in a position to investigate this crucial issue.

Our calculations will examine $A B$ diblock copolymers, which represent a model system for block copolymers as well as lyotropic liquid crystals in general. They are simple linear polymer chains consisting of $f N A$-type segments joined to $(1-f) N B$-type segments, where $N$ represents the total molecular weight and $f$ specifies the composition of the molecule. (We follow the convention of defining segments with a common volume of $\rho_{0}^{-1}$.) The theoretical mean-field phase diagram for monodisperse diblock melts (i.e., liquids) is shown in Fig. 1(a). When the incompatibility between the unlike segments, as measured by the Flory-Huggins $\chi$ interaction parameter, becomes sufficient, the disordered melt self-assembles into a periodically ordered structure with nanosized domains. Relatively symmetric diblocks create alternating lamellar $(L)$ domains, while highly asymmetric diblocks form cylindrical $(C)$ or spherical $(S)$ domains containing the shorter minority blocks. At intermediate asymmetries, two complex phases compete for stability: the bicontinuous gyroid $(G)$ where the minority domains form two intertwining threefold coordinated networks, and the perforated-lamellar $(P L)$ where the minority domains form a stack of perforated lamellae. $P L$ is slightly less stable than $G$, and is therefore absent from the equilibrium phase diagram. The agreement of the theoretical diagram with experiment is remarkably good $[6,16]$, apart from the immediate region along the order-disorder transition (ODT) where the weakly segregated structures are disordered by thermal fluctuations [12,17].

The physics behind this behavior is amazingly simple [16]; for the most part, it just involves the tension of the internal interfaces between the $A$ - and $B$-rich domains and an effective domain elasticity due to the entropy loss of extending the $A$ and $B$ blocks away from the interface. The competition between the interfacial tension and the entropic stretching energy dictates the equilibrium periodicity of the morphology. An asymmetry in the lengths of the $A$ and $B$ blocks causes an imbalance in the elasticity of their respective domains, which induces a spontaneous curvature in the interface; this accounts for the progression of phases across the diagram in Fig. 1(a). Further details are then explained in terms of two competing tendencies: interfacial tension favors the formation of constant- 

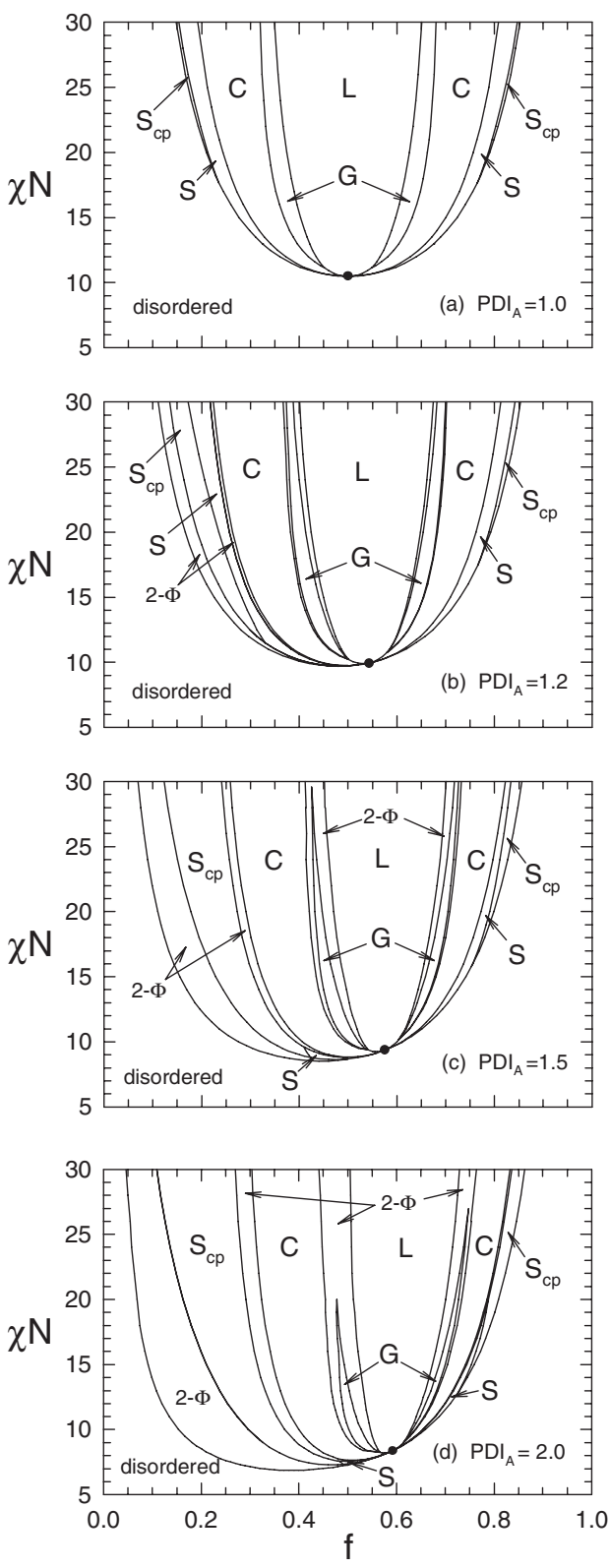

FIG. 1. Phase diagrams for $A B$ diblocks with various levels of $A$-block polydispersity, where $N$ is the number-averaged polymerization index and $f$ is the overall volume fraction of $A$ segments. All the single-phase regions are identified, but only the larger two-phase $(2-\Phi)$ regions are labeled.

curvature interfaces, while the entropic elasticity favors domains of uniform thickness. The inability of a structure to simultaneously accommodate both tendencies is referred to as packing frustration. Minimization of packing frustration causes the cylinders to arrange hexagonally and the spheres to adopt a bcc lattice. Self-consistent field theory (SCFT) does predict a narrow $S_{\mathrm{cp}}$ region along the ODT where the spheres become close-packed (either fcc or hcp), but this happens because the frustration is relieved by a small population of diblocks that swells the matrix by dislodging from the spherical domains. In experiments, this reduction in packing frustration results in a weak association between the spheres, allowing thermal fluctuations to destroy the crystalline order transforming $S_{\mathrm{cp}}$ into a liquidlike micellar region. The complex $G$ and $P L$ phases possess particularly large degrees of packing frustration, and $P L$ is metastable simply because it has slightly more. This thorough intuitive understanding of the monodisperse system will be invaluable when it comes time to explain our polydispersity effects.

Polydispersity is gauged by the ratio, $\mathrm{PDI} \equiv\left\langle N_{w}\right\rangle /\left\langle N_{n}\right\rangle$, of the weight- and number-averaged molecular weights. This index is one for a perfectly monodisperse system, and typically ranges up to 1.1 for the narrow distributions synthesized by living anionic polymerization. The newer controlled radical polymerizations generally result in indices of 1.2-1.4 [5], which can easily become 2 or more [4]. For the sake of simplicity, we will assume monodisperse $B$ blocks and allow the $A$ blocks to have $f N \sigma$ segments, where $\sigma$ is a random variable with the usual SchulzZimm distribution [1,7,12-15],

$$
p(\sigma)=k^{k} \sigma^{k-1} \exp (-k \sigma) / \Gamma(k),
$$

for which $\mathrm{PDI}_{A}=(k+1) / k$. From here on, $N$ represents the number-averaged molecular-weight of the diblocks and $f$ is the overall fraction of $A$ segments.

The SCFT [16] used for diblock copolymer melts treats the molecules as thin elastic threads and replaces the molecular interactions acting on $A$ and $B$ segments by the mean fields,

$$
\begin{aligned}
& w_{A}(\mathbf{r})=\chi N \phi_{B}(\mathbf{r})+\xi(\mathbf{r}), \\
& w_{B}(\mathbf{r})=\chi N \phi_{A}(\mathbf{r})+\xi(\mathbf{r}),
\end{aligned}
$$

respectively. Here $\phi_{\alpha}(\mathbf{r})$ is the dimensionless concentration of $\alpha$-type segments and $\xi(\mathbf{r})$ is a Lagrange multiplier field used to enforce the incompressibility constraint,

$$
\phi_{A}(\mathbf{r})+\phi_{B}(\mathbf{r})=1 .
$$

This mean-field treatment just requires the statistical mechanics of a single molecule in the external fields, $w_{\alpha}(\mathbf{r})$, which is done by calculating two partition functions for a diblock with $s_{A} N A$ segments and $s_{B} N B$ segments. The first, $q\left(\mathbf{r}, s_{A}, s_{B}\right)$, is for the molecule with its $A$ end fixed at $\mathbf{r}$, and is evaluated by starting with $q(\mathbf{r}, 0,0)=1$ and integrating

$$
\frac{\partial}{\partial s} q(\mathbf{r}, s, 0)=\left[\frac{a^{2} N}{6} \nabla^{2}+w_{A}(\mathbf{r})\right] q(\mathbf{r}, s, 0)
$$

from $s=0$ to $s_{A}$, and then integrating

$$
\frac{\partial}{\partial s} q\left(\mathbf{r}, s_{A}, s\right)=\left[\frac{a^{2} N}{6} \nabla^{2}+w_{B}(\mathbf{r})\right] q\left(\mathbf{r}, s_{A}, s\right)
$$

from $s=0$ to $s_{B}$. The other, $q^{\dagger}\left(\mathbf{r}, s_{A}, s_{B}\right)$, is for the molecule with its $B$ end fixed at $\mathbf{r}$; this time one starts with $q^{\dagger}(\mathbf{r}, 0,0)=1$, but integrates $q^{\dagger}(\mathbf{r}, 0, s)$ from $s=0$ to $s_{B}$ using Eq. (6), and then integrates $q^{\dagger}\left(\mathbf{r}, s, s_{B}\right)$ from $s=0$ to $s_{A}$ using Eq. (5). 
In a single-phase region, the statistical mechanics can be performed with the canonical ensemble [18], in which the concentrations are evaluated using

$$
\begin{aligned}
\phi_{A}(\mathbf{r})= & \int_{0}^{\infty} d \sigma p(\sigma) \frac{\mathcal{V}}{\mathcal{Q}(\sigma)} \\
& \times \int_{0}^{f \sigma} d s q(\mathbf{r}, s, 0) q^{\dagger}(\mathbf{r}, f \sigma-s, 1-f), \\
\phi_{B}(\mathbf{r})= & \int_{0}^{\infty} d \sigma p(\sigma) \frac{\mathcal{V}}{\mathcal{Q}(\sigma)} \\
& \times \int_{0}^{1-f} d s q(\mathbf{r}, f \sigma, s) q^{\dagger}(\mathbf{r}, 0,1-f-s),
\end{aligned}
$$

where $\mathcal{V}$ is the total volume of the melt and

$$
\mathcal{Q}(\sigma)=\int d \mathbf{r} q(\mathbf{r}, f \sigma, 1-f)
$$

is the single-chain partition function for an unconstrained diblock with $f \sigma N A$ segments and $(1-f) N B$ segments. Using these expressions for $\phi_{\alpha}(\mathbf{r})$, we adjust the fields so as to satisfy the self-consistent conditions, Eqs. (2)-(4), while at the same time we vary the periodicity of the morphology so as to minimize the canonical free energy,

$$
\begin{aligned}
\frac{N F_{c}^{C}}{k_{B} T \rho_{0} \mathcal{V}}= & \int_{0}^{\infty} d \sigma p(\sigma)\left[\ln \left(\frac{p(\sigma) \mathcal{V}}{\mathcal{Q}(\sigma)}\right)-1\right] \\
& -\frac{1}{\mathcal{V}} \int d \mathbf{r}\left[\chi N \phi_{A}(\mathbf{r}) \phi_{B}(\mathbf{r})+\xi(\mathbf{r})\right] .
\end{aligned}
$$

The edge of a single-phase region (i.e., cloud point) occurs when it becomes favorable to nucleate an infinitesimal amount of another phase. While the majority (cloud) phase must maintain the parent distribution $p(\sigma)$, the new minority (shadow) phase is free to adopt any molecularweight distribution. However, equilibrium with the cloud phase imposes a specific chemical potential,

$$
\mu(\sigma)=-k_{B} T \ln \left(\frac{p(\sigma) \mathcal{V}}{\mathcal{Q}(\sigma)}\right),
$$

for each molecular weight (i.e., $\sigma$ ). Therefore, the shadow phase must be solved in the grand-canonical ensemble [19], in which the concentrations are given by equivalent expressions to Eqs. (7) and (8), but with the substitution,

$$
p(\sigma) \frac{\mathcal{V}}{\mathcal{Q}(\sigma)} \rightarrow z(\sigma) \equiv \exp \left(-\frac{\mu(\sigma)}{k_{B} T}\right) .
$$

As before, the fields are adjusted to satisfy the same selfconsistent conditions, and the domain size is selected to minimize the grand-canonical free energy,

$$
\begin{aligned}
\frac{N F_{\mathrm{gc}}^{S}}{k_{B} T \rho_{0} \mathcal{V}}= & -\int_{0}^{\infty} d \sigma z(\sigma) \frac{\mathcal{Q}(\sigma)}{\mathcal{V}} \\
& -\frac{1}{\mathcal{V}} \int d \mathbf{r}\left[\chi N \phi_{A}(\mathbf{r}) \phi_{B}(\mathbf{r})+\xi(\mathbf{r})\right] .
\end{aligned}
$$

To compare the stability of the two phases, we convert the canonical free energy of the cloud phase to the grandcanonical one using

$$
\begin{aligned}
\frac{N F_{\mathrm{gc}}^{C}}{k_{B} T \rho_{0} \mathcal{V}} & \equiv \frac{N F_{c}^{C}}{k_{B} T \rho_{0} \mathcal{V}}-\int_{0}^{\infty} d \sigma p(\sigma) \frac{\mu(\sigma)}{k_{B} T}, \\
& =-1-\frac{1}{\mathcal{V}} \int d \mathbf{r}\left[\chi N \phi_{A}(\mathbf{r}) \phi_{B}(\mathbf{r})+\xi(\mathbf{r})\right] .
\end{aligned}
$$

The cloud curve occurs at the first point where a solution exists for which $F_{\mathrm{gc}}^{S}=F_{\mathrm{gc}}^{C}$. The numerical evaluation of SCFT is made feasible for large polydispersities by adapting the new algorithm in Ref. [15].

Figure 1 illustrates the effect of $A$-block polydispersity on the equilibrium phase diagram. The shift in the phase boundaries towards large $f$ has already been observed in several experiments $[5,7,8]$, and predicted by an earlier SCFT calculation [13]. The explanation for this shift is simple; the polydispersity reduces the entropic elasticity of the $A$ domains [15], which causes a tendency for the interface to curve towards them. Naturally the reduction in $A$-domain elasticity also increases the periodicity of the morphologies, but this too has already been demonstrated by SCFT $[13,15]$ and likewise confirmed by experiments [7,9].

Previous calculations $[12,13]$ have only considered the $L, C, G$, and $S$ structures, whereas we have also examined $P L$ and $S_{\mathrm{cp}} . P L$ has been observed [8] in polydisperse melts, but it was only thought to be metastable; indeed, this conclusion agrees with our calculation. On the other hand, the stability of $S_{\mathrm{cp}}$ is significantly enhanced by polydispersity. This is because it reduces the packing frustration that favors bcc spheres, but in two different ways. At small $f$ where the polydispersity is in the minority domain, there will be a greater population of diblocks that become dislodged from the interface (primarily those with the shorter $A$ blocks) free to fill the interstitial regions in the matrix responsible for packing frustration. At large $f$ where the matrix blocks are polydisperse, the packing frustration will be relieved by the availability of long $A$ blocks capable of filling the interstitial regions without excessive stretching. With the reduction in packing frustration, the free energy difference among alternative crystal arrangements becomes negligible, and therefore thermal fluctuations should disrupt long-range order; this is consistent with the liquidlike disorder of spheres observed in experiments $[4,5]$. Furthermore, polydispersity should enhance the fluctuation-induced shift of the ODT towards higher $\chi N$ [17] countering the mean-field shift towards lower $\chi N$ predicted in Fig. 1; again this is consistent with recent experiments [8].

Our principal aim was to examine whether or not there are significant two-phase (2- $\Phi)$ regions, and indeed there are. They occur due to fractionation of the molecular weights, and were not predicted in the earlier SCFT calculation of Cooke and Shi [13] because they assumed the parent distribution $p(\sigma)$ for all phases. Here each shadow 
phase emerges with its own distinct distribution $p^{S}(\sigma)=$ $z(\sigma) Q(\sigma) / \mathcal{V}$ favoring its preferred interfacial curvature. At $L+C$ coexistence, for example, $L$ preferentially selects the more symmetric diblocks, while $C$ takes on the more asymmetric molecules. Although all single-phase regions are separated by coexistence as soon as there is any polydispersity, the $2-\Phi$ windows are initially very narrow. They widen as $\mathrm{PDI}_{A}$ increases gradually occupying a significant portion of the phase diagram by $\mathrm{PDI}_{A}=2.0$. There is, however, a clear asymmetry; the windows are much larger when the minority domain is polydisperse (i.e., small $f$ ). The $S_{\mathrm{cp}}+S$ region is an exception; the two phases are so similar that there is no advantage to fractionation and thus the 2- $\Phi$ window remains narrow regardless of which domain is polydisperse.

The complex $G$ phase is known to possess high levels of packing frustration, but it nevertheless exists between $L$ and $C$ because of its intermediate interfacial curvature [16]. One might think that polydispersity would relieve some of this frustration enhancing the size of the $G$ region, but fractionation of $p(\sigma)$ proves to be the more favorable option. The advantage of $L+C$ coexistence is that $C$ has only a tiny amount of packing frustration while $L$ has absolutely none [16]. Fractionation does, however, reduce the translational entropy of the molecules, which becomes relatively important at lower segregations; this explains why $G$ remains stable at small $\chi N$. Not only are the explanations simple, but also the SCFT predictions are in agreement with the repeated observation of $G$ at $\chi N \sim 10$ $[7,8]$ and $L+C$ coexistence at $\chi N \geqslant 50[4,10]$. Note that $L+C$ coexistence also occurs in polydisperse $A B A$ triblock melts at strong segregations [11].

The reduction in packing frustration and occurrence of macrophase separation will be of some concern for the new controlled radical polymerizations [4,5], but fortunately Figs. 1(b) and 1(c) indicate that the problem will not be too serious if the polydispersity does not get out of control. However, one must remember that the effects will be greater when both blocks are polydisperse and may be more significant for other architectures [11]. Nevertheless, there are bound to be simple ways of reducing the undesirable effects by optimizing the system parameters or perhaps by modifying the shape of $p(\sigma)$. SCFT will certainly provide a valuable tool for investigating these possibilities. If the macrophase separation and disorder can be tamed, there will be a tremendous opportunity for economical block-copolymer materials.

We can also expect that, to some degree, the polydispersity effects for diblock copolymer melts will carry over to conventional lyotropic liquid crystals involving aqueous solutions of lipids or surfactants. After all, they exhibit analogous phases and their behavior is again explained in terms of packing frustration [20]. There may not be the same shift in the phase boundaries or increase in domain spacing, because lipids and surfactants do not have nearly as much conformational entropy as polymers. However, the polydispersity should still relieve packing frustration, causing a reduction in long-range order. We could also expect polydispersity to favor $L+C$ coexistence over the complex bicontinuous phases [21]. Such results will also have an impact on biology, given that these morphologies are commonly observed in living cells [22]. Polydispersity may not be of great practical importance in synthetic systems because of the relative ease of producing monodisperse lipids, but it certainly will be in biological cells where polydispersity is rampant.

We are grateful to Marc Hillmyer, Nate Lynd, John Seddon, and Richard Templer for useful discussions and to the EPSRC (No. EP/E010342/1) for financial support.

[1] P. Sollich, J. Phys. Condens. Matter 14, R79 (2002).

[2] P. Sollich and M.E. Cates, Phys. Rev. Lett. 80, 1365 (1998); P. B. Warren, ibid. 80, 1369 (1998); 80, 3671 (1998).

[3] A. M. Bohle, R. Holyst, and T. Vilgis, Phys. Rev. Lett. 76, 1396 (1996); F. M. van der Kooij, K. Kassapidou, and H. N. W. Lekkerkerker, Nature (London) 406, 868 (2000); A. V. Petukhov et al., Phys. Rev. Lett. 95, 077801 (2005).

[4] D. Bendejacq et al., Macromolecules 35, 6645 (2002).

[5] A.-V. Ruzette et al., Macromolecules 39, 5804 (2006).

[6] F. S. Bates and G. H. Fredrickson, Phys. Today 52, No. 2, 32 (1999).

[7] N. A. Lynd and M. A. Hillmyer, Macromolecules 38, 8803 (2005).

[8] N. A. Lynd and M. A. Hillmyer, Macromolecules (to be published).

[9] Y. Matsushita et al., Macromolecules 36, 8074 (2003).

[10] N. A. Lynd, B. D. Hamilton, and M. A. Hillmyer, J. Polym. Sci. B (to be published); the two macrophase-separated samples are consistent with $L+C$ coexistence (private communication).

[11] A. Noro, et al., Macromolecules 37, 3804 (2004).

[12] C. Burger, W. Ruland, and A. N. Semenov, Macromolecules 23, 3339 (1990); 24, 816 (1991).

[13] D. M. Cooke and A.-C. Shi, Macromolecules 39, 6661 (2006).

[14] S. W. Sides and G. H. Fredrickson, J. Chem. Phys. 121, 4974 (2004).

[15] M. W. Matsen, Eur. Phys. J. E 21, 199 (2006).

[16] M. W. Matsen, J. Phys. Condens. Matter 14, R21 (2002).

[17] G. H. Fredrickson and H. Helfand, J. Chem. Phys. 87, 697 (1987).

[18] M. W. Matsen and M. Schick, Phys. Rev. Lett. 72, 2660 (1994).

[19] M. W. Matsen, Phys. Rev. Lett. 74, 4225 (1995).

[20] P. M. Duesing, R. H. Templer, and J. M. Seddon, Langmuir 13, 351 (1997).

[21] G. C. Shearman et al., Langmuir 23, 7276 (2007).

[22] Z. A. Almsherqi, S. D. Kohlwein, and Y. Deug, J. Cell Biol. 173, 839 (2006). 